\title{
Attenuation of COVID-19-induced cytokine storm in a young male patient with severe respiratory and neurological symptoms
}

\author{
Christian Muschitz (D) - Anita Trummert · Theresa Berent · Norbert Laimer · Lukas Knoblich · Gerd Bodlaj • \\ Alexander Krainer · Christoph Linder · Heinrich Resch
}

Received: 23 January 2021 / Accepted: 27 March 2021 / Published online: 27 April 2021

(C) The Author(s) 2021

\begin{abstract}
Summary Severe acute respiratory syndrome coronavirus type 2 (SARS-CoV-2), the etiological agent of coronavirus disease 2019 (COVID-19), produces protean manifestations and causes indiscriminate havoc in multiple organ systems. This rapid and vast production of proinflammatory cytokines contributes to a condition termed cytokine storm. A 35-year-old, otherwise healthy, employed, male patient was tested positive for COVID-19. He was admitted to the hospital on disease day 10 due to retarded verbal reactions and progressive delirium. On account of these conditions and the need for noninvasive/invasive ventilation, a combination treatment with baricitinib and remdesivir in conjunction with standard of care was initiated. The cytokine storm was rapidly blocked, leading to a vast pulmonary recovery with retarded recovery of the central nervous system. We conclude that the rapid blockade of the COVID-19-induced cytokine storm should be considered of avail as a principle of careful decision-making for effective recovery.
\end{abstract}

Assoc. Prof. C. Muschitz, M.D. (西) · T. Berent · N. Laimer ·

L. Knoblich · G. Bodlaj · H. Resch

Medical Department II-the VINFORCE Study

Group, Academic Teaching Hospital of the Medical

University of Vienna, St. Vincent Hospital Vienna,

Stumpergasse 13, 1060 Vienna, Austria

christian.muschitz@bhs.at

\section{A. Trummert · C. Linder}

Department of Neurology, Divine Savior Hospital Vienna,

Dornbacher Straße 20-30, 1170 Vienna, Austria

\section{A. Krainer}

Department of Anesthesiology, St. Vincent Hospital Vienna, Stumpergasse 13, 1170 Vienna, Austria

\section{H. Resch}

Medical Faculty of Bone Diseases, Sigmund Freud University, Freudplatz 1, 1170 Vienna, Austria
Keywords COVID-19 - Cytokine storm - Baricitinib • Remdesivir · Inflammation

\section{Introduction}

Severe acute respiratory syndrome coronavirus type 2 (SARS-CoV-2), the etiological agent of coronavirus disease 2019 (COVID-19), produces protean manifestations and causes indiscriminate havoc in multiple organ systems, in particular the lungs, heart, brain, bone, kidneys and vasculature. Cytokines such as interleukin (IL)-1a, IL-1b, IL-6, and tumor necrosis factor alpha, contribute critically to normal host defences but when produced inappropriately or in excess, they perturb all of the protective functions of the normal endothelium and potentiate pathological processes. This rapid and vast production of proinflammatory cytokines contributes to a condition termed cytokine storm. Induction of IL- 6 production by IL-1 provides an amplification loop [1].

In a crisis scenario with limited therapeutic resources, patient care often involves management in the ward by means of noninvasive ventilation or highflow nasal oxygen. Based on clinical judgment or recommendations by the World Health Organization and national authorities, treatment for critically ill patients includes a combination of antiviral (e.g., remdesivir) and anti-inflammatory (e.g., dexamethasone) drugs in an attempt to delay or avoid mechanical ventilation in the intensive care unit.

Janus kinase (JAK) inhibitors, particularly baricitinib, are currently used in the treatment of rheumatoid arthritis. Baricitinib has been proven to interrupt the passage and intracellular assembly of SARSCoV-2 into target cells and thereby to disrupt inflammation [2, 3]. Furthermore, in patients with COVID19 , the combination of baricitinib and remdesivir was 
recently evaluated to have favorable effects on clinical improvements and recovery time [4].

In this short report, we present the results of the aforementioned combination in a young male patient with severe respiratory and neurological symptoms.

\section{Patient description}

A 35-year-old, otherwise healthy, employed, male Caucasian patient was tested positive for COVID19. Initially asymptomatic, abdominal symptoms emerged. He had two medical visits during self-isolation. Metoclopramide was administered for 5 days orally and once intravenously (disease day 8). On disease day 10 (22 December 2020), he was admitted to the Medical Department II at St. Vincent Hospital in Vienna due to retarded verbal reactions and progressive delirium. The cycle threshold (Ct) value was $>30$. Vital signs and oxygen saturation, with the exception of mild sinus tachycardia (110/min), were within normal range and no fever was detected. An acute cranial computed tomography showed a symmetric calcification of basal ganglia, the serum values showed normal calcium metabolism and no signs of hyperparathyroidism, hypoparathyroidism or pseudohypoparathyroidism. The $\mathrm{Ct}$ value was above 30 , the initial $\mathrm{X}$-ray of the chest revealed small, symmetrically spotted infiltrates. After neurological consultation, the patient received intravenous crystalloid liquids and oral risperidone $1.0 \mathrm{mg}$ /day (duration 10 days) with the primary diagnosis of an incipient delirium caused by SARS-CoV-2. Based on the chest X-ray, a mild oxygen supplementation $(1 \mathrm{~L} / \mathrm{min})$ was also administered together with anticoagulants and cholecalciferol. The patient had a rapid recovery (within $2 \mathrm{~h}$ ) of all clinical symptoms and remained stable for the following $30 \mathrm{~h}$. Subsequently, on admission day 3 the respiratory and neurological situations deteriorated and the patient

Fig. 1 Chest X-ray images at different time points of treatment
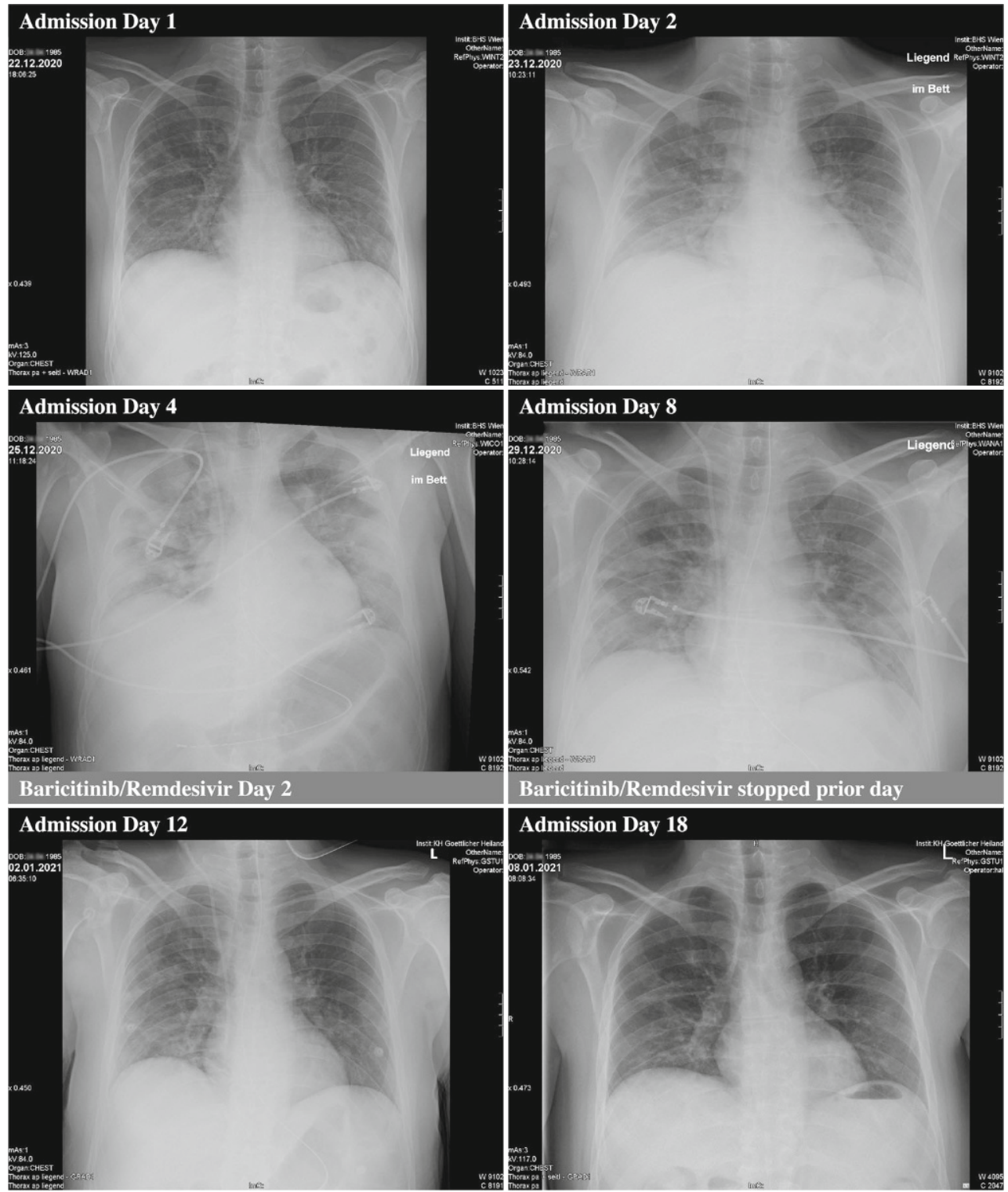


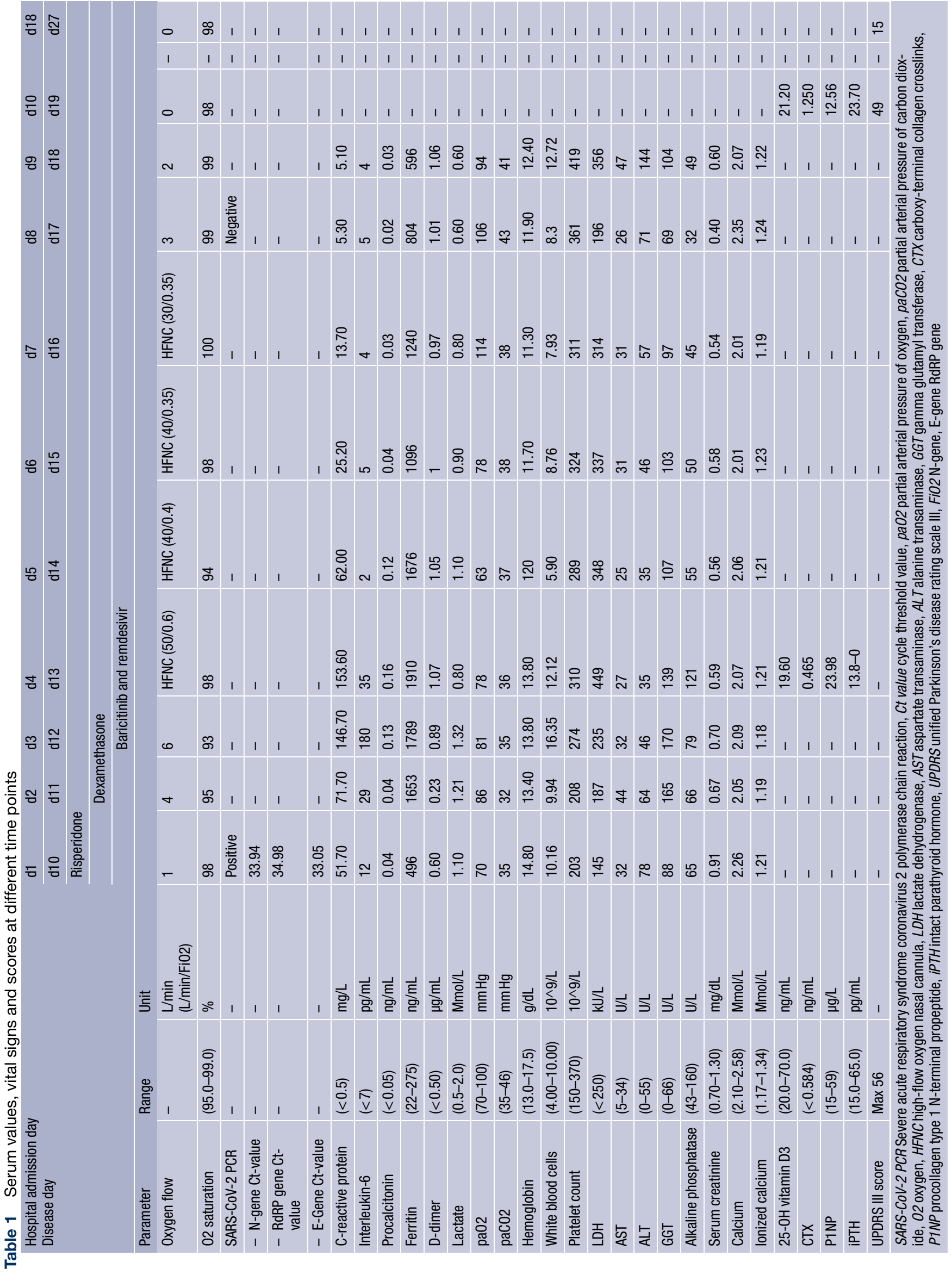


developed fever and needed noninvasive oxygen ventilation. Movement disorders and inadequate verbal reactions evolved. Treatment was therefore amplified with dexamethasone ( $6 \mathrm{mg} /$ day, duration 7 days) and ceftriaxone ( $2 \mathrm{~g} /$ day, duration 8 days). The subsequent blood cultures were negative and no coinfections were detected during the course of treatment. At this point, the serum levels of C-reactive protein (CRP) increased from initially $50 \mathrm{mg} / \mathrm{L}$ to $146 \mathrm{mg} / \mathrm{L}$ and of procalcitonin from $<0.05$ to $0.13 \mathrm{ng} / \mathrm{mL}$. Due to highly elevated IL-6 (180 pg/mL) on disease day 12 and after careful evaluation, a combination treatment was initiated with $4 \mathrm{mg}$ oral baricitinib (duration 5 days) and $200 \mathrm{mg}$ intravenous remdesivir (day 1, reduction to $100 \mathrm{mg}$ on the subsequent day, total duration 5 days). Due to an unsatisfactory and continuous decline of peripheral oxygen saturation, the patient received oxygen ventilation up to a limit of $6 \mathrm{~L} / \mathrm{min}$. Communication deteriorated with intermittent disorientation and $12 \mathrm{~h}$ later, with new and ongoing tachypnea and decreasing oxygen saturation values, the patient was transferred to the intermediate care unit (IMCU) for high-flow oxygen supplementation (high-flow oxygen nasal cannula, HFNC. range $40-50 \mathrm{~L} / \mathrm{min}$, fraction of inspired oxygen, FiO2, 0.4-0.6). The patient needed HFNC for a total of $75 \mathrm{~h}$ and received oxygen supplementation for a consecutive day. A hypokinetic-rigid syndrome based on a COVID-19 encephalopathy was diagnosed at this point in time.

After initiation of the combination treatment with baricitinib and remdesivir in conjunction with dexamethasone, the elevated IL-6 levels normalized within less than $48 \mathrm{~h}$, the CRP levels were within normal range after 9 days, and consecutive chest X-rays showed an improvement within 6 days. Inflammatory cytokines remained decreased and within normal range and ferritin remained increased. Bone metabolism showed an isolated increase in osteoclast activity (CTX levels) and the elevated liver enzymes slowly declined (Table 1; Fig. 1).

After 11 days at the Medical Department II (including 5 days at the IMCU), the patient was transferred to the Department of Neurology at the partner Hospital of the Divine Savior in Vienna, Austria for further treatment work-up and treatment of ongoing extrapyramidal symptoms. On admission the patient presented with a pronounced hypomimia, rigidity, bradykinesis, tremor and a mild tetraparesis of the extremities as well as dysarthria and dysphagia, resulting in a unified Parkinson's disease rating scale (UPDRS) III score of 49 (out of possible 56). Risperidone was terminated 2 days before and the family already reported some improvement of speech. Magnetic resonance imaging (MRI) of the brain, a lumbar puncture and an electroencephalogram (EEG) were performed. The MRI revealed no structural alteration of the brain apart from the previously shown basal ganglia calcifications. All cerebrospinal fluid parameters showed no signs of viral or bacterial dis- ease or inflammation, the EEG was normal. Based on the interdisciplinary treatment, the patient recovered rapidly. The initially started levodopa (L-DOPA) treatment (maximum $50 \mathrm{mg}$ tid) was tapered without negative effect. After 14 days under neurological treatment and intensive physiotherapy he was fully orientated, ambulating freely and had normal vital signs and serum values. Slight movement disorders are still detectable, but minor compared to the severity of symptoms at the time point at the admission to the hospital. The UPDRS III score improved to 15 and the patient was discharged from the hospital. Rehabilitation and muscle perseverance training is needed. One month after the first symptoms of the COVID-19 infection a slight reduction in retentiveness is reported by the family.

\section{Discussion}

We report a case of a young patient with COVID-19associated, initially mild abdominal symptoms and progressive severe respiratory and neurological symptoms induced by a cytokine storm. Proinflammatory cytokines conspire to elicit from endothelial cells a change from their homeostatic functions to those that can contribute to thrombosis and local tissue injury [1]. In line with WHO recommendations, the standard of care in symptomatic patients includes oxygen supplementation and dexamethasone. The deterioration of clinical symptoms and serological findings demands a therapeutic boost on account of the pathophysiology of this novel disease. Based on recently published data from a double-blind, placebocontrolled trial with a comparable study population, this young and otherwise healthy patient received an additional combination of baricitinib and remdesivir [4]. The administered and recently published combination treatment was safe and immediately lowered elevated levels of IL- 6 and other inflammatory cytokines. It probably reduced the duration at the IMCU with HFNC and led to the patient's recovery with reduction symptoms of the affected organ systems.

Serological, radiological and clinical findings suggest the need for an individual and targeted approach. The decision of treatment enhancement beyond dexamethasone and oxygen with different RCT-proven approaches to reduce the cytokine storm was based on the patient's rapid pulmonary and neurological worsening in an attempt to avert the use of mechanical ventilation.

The potential role of baricitinib in the treatment of COVID-19 is based on artificial intelligence algorithms. A potent JAK $1 / 2$ inhibitor would be able to directly mitigate the inflammatory response/cytokine storm triggered by the COVID-19 infection. This drug has a high affinity for protein kinases directly involved in the endocytosis of coronaviruses $[5,6]$. Disease severity is marked by hyperinflammation and 
cytokine storm syndromes driven by IL-6 expression through the JAK signal transducers and activators of transcription pathway [7].

Despite concerns about immunosuppression, secondary infections, or increased risk of thrombosis, the administration of baricitinib in conjunction with dexamethasone or antiviral agents in clinical trials has not been associated with a significantly higher incidence of adverse events. In contrast, the JAK inhibitor baricitinib, with a half-life of $12 \mathrm{~h}$ in combination with remdesivir, exerts beneficial effects in terms of the time of oxygen supplementation and the time to recovery. These effects were also observed in this patient and corroborated by numerous serological, radiological and neurological investigations during treatment [4-7].

Besides the pulmonary affection, the patient had also developed delirium and an extrapyramidal syndrome (EPS). Neurological symptoms are common in COVID-19, including anosmia and ageusia, non-specific symptoms, such as dizziness and headache, and severe conditions, such as ischemic stroke, hemorrhagic and/or reversible encephalopathy syndrome with epileptic seizures. Extrapyramidal signs in patients with encephalopathy have been reported. Ischemic and hemorrhagic strokes seem to have a higher incidence in patients with a severe COVID-19 course of disease. The two major competing hypotheses are based on neurotropism and direct invasion of SARS-CoV-2 into the central nervous system (CNS), and indirect mechanisms mediated by the cytokine storm induced by systemic SARS-CoV-2 infection. Post-mortem examinations of CNS in this patient population have demonstrated mild neuropathological changes, with pronounced neuroinflammatory changes in the brainstem being the most common finding [8].

In this context it remains unclear if risperidone, which was started due to the delirium contributed to the EPS on the basis of the bilateral calcification of the basal ganglia or if the EPS was part of an encephalopathy syndrome due to COVID-19 infection. To date, evidence in the literature is scarce, although the use of this antipsychotic drug has been proven to be efficacious in this specific context $[9,12]$. The dopamine agonist metoclopramide, which was administered prior to hospital admission, is able to intensify extrapyramidal symptoms in patients with Parkinson's disease. While no adverse events in COVID-19 patients have been published to date, metoclopramide was recently even identified as a protective factor [10].

The SARS-CoV-2 also causes an activation of nuclear factor kappa-light-chain-enhancer of activated B-cells $(\mathrm{NF}-\kappa \mathrm{B})$ with subsequent bone resorption through direct activation of the receptor activator of NF- $\kappa \mathrm{B}$ ligand (RANK-RANKL) system. Recent data suggest that this disease has the potential to act directly on bone resorption units with unfavorable long- term effects on bone metabolism (uncoupling) and a possibly increased risk of fragility fractures [11].

A multifactorial therapeutic approach with immunomodulatory drugs, based on clinical findings and trials, may help to achieve therapeutic goals. We conclude that the rapid blockade of the COVID-19induced cytokine storm should be considered of avail as a principle of careful decision-making for effective recovery while requiring long-term surveillance of COVID-19 patients.

Acknowledgements The authors acknowledge the work of all nurses and the laboratory staff at both hospitals.

Author Contribution All authors contributed to the study conception and design. The first draft of the manuscript was written by Christian Muschitz and all authors commented on previous versions of the manuscript. All authors read and approved the final manuscript.

Funding Open access funding provided by Medical University of Vienna.

Conflict of interest C. Muschitz, A. Trummert, T. Berent, N. Laimer, L. Knoblich, G. Bodlaj, A. Krainer, C. Linder and $\mathrm{H}$. Resch declare that they have no competing interests.

Open Access This article is licensed under a Creative Commons Attribution 4.0 International License, which permits use, sharing, adaptation, distribution and reproduction in any medium or format, as long as you give appropriate credit to the original author(s) and the source, provide a link to the Creative Commons licence, and indicate if changes were made. The images or other third party material in this article are included in the article's Creative Commons licence, unless indicated otherwise in a credit line to the material. If material is not included in the article's Creative Commons licence and your intended use is not permitted by statutory regulation or exceeds the permitted use, you will need to obtain permission directly from the copyright holder. To view a copy of this licence, visit http://creativecommons.org/licenses/by/4.0/.

\section{References}

1. Libby P, Lüscher T. COVID-19 is, in the end, an endothelial disease. Eur Heart J.2020;41(32):3038-3034.

2. Seif F, Aazami H, Khoshmirsafa M, Kamali M, Mohsenzadegan M, Pornour M, et al. JAK inhibition as a new treatment strategy for patients with COVID-19. Int Arch Allergy Immunol. 2020;181(6):467-465.

3. Rodríguez Y, Novelli L, Rojas M, De Santis M, AcostaAmpudia Y, Monsalve DM, et al. Autoinflammatory and autoimmune conditions at the crossroad of COVID-19. JAutoimmun. 2020;114:102506.

4. Kalil AC, Patterson TF, Mehta AK, Tomashek KM, Wolfe CR, Ghazaryan V, et al. Baricitinib plus Remdesivir for hospitalized adults with Covid-19. N Engl J Med. 2020;11:NEJMoa2031994.

5. Richardson P, Griffin I, Tucker C, Smith D, Oechsle O, Phelan A, et al. Baricitinib as potential treatment for 2019-nCoV acute respiratory disease. Lancet. 2020;395(10223):e30-el.

6. Stebbing J, Krishnan V, de Bono S, Ottaviani S, Casalini G, Richardson PJ, et al. Mechanism of baricitinib supports artificial intelligence-predicted testing in COVID-19 patients. EMBO Mol Med. 2020;12(8):e12697.

7. Mehta P, Cron RQ, Hartwell J, Manson JJ, Tattersall RS. Silencing the cytokine storm: the use of intravenous 


\section{short report}

anakinra in haemophagocytic lymphohistiocytosis or macrophage activation syndrome. Lancet Rheumatol. 2020;2(6):e358-e67.

8. Matschke J, Lütgehetmann M, Hagel C, Sperhake JP, Schröder AS, Edler C, et al. Neuropathology of patients with COVID-19 in Germany: a post-mortem case series. Lancet Neurol. 2020;19(11):919-919.

9. Demir G, Balaban O, Tekeci MH, Issı Z, Erdem AF. Fahr's syndrome presenting with seizures in SARS-CoV-2 (COVID-19) pneumonia-a case report. Neurol Sci. 2020;41(11):3063-5. https://doi.org/10.1007/s10072-020-04733-7.

10. Sen A. Repurposing prolactin as a promising immunomodulator for the treatment of COVID-19: are common antiemetics the wonder drug to fight coronavirus? Med Hypotheses. 2020;144:110208.
11. Salvio G, Gianfelice C, Firmani F, Lunetti S, Balercia G, Giacchetti G. Bone metabolism in SARS-coV-2 disease: possible osteoimmunology and gender implications. Clin Rev Bone Miner Metab. 2020; https://doi.org/10.1007/ s12018-020-09274-3.

12. Ostuzzi G, Gastaldon C, Papola D, Fagiolini A, Dursun S, Taylor D, et al. Pharmacological treatment of hyperactive delirium in people with COVID-19: rethinking conventional approaches. Ther Adv Psychopharmacol. 2020;10:2045125320942703. https://doi.org/10.1177/ 2045125320942703.

Publisher's Note Springer Nature remains neutral with regard to jurisdictional claims in published maps and institutional affiliations. 